\title{
Estimating Optimum Overrelaxation Parameters
}

\author{
By L. A. Hageman and R. B. Kellogg*
}

1. Introduction. In using the successive overrelaxation (S.O.R.) iteration method to solve the matrix equation

$$
A \mathrm{x}=\mathrm{f},
$$

finding the optimum overrelaxation parameter $\omega_{b}$ is an important and often a difficult part of the problem [1, p. 257]. When $A$ is a 2-cyclic, consistently ordered, real symmetric, positive-definite matrix (this will be assumed in the rest of the paper), only the spectral radius $\rho\left(\mathscr{L}_{1}\right)$ of the associated Gauss-Seidel iteration matrix $\mathfrak{L}_{1}$ is needed to determine the optimum parameter $\omega_{b}\left[2\right.$, p. 109]. When $\rho\left(\mathscr{L}_{1}\right)$ is close to unity, small changes in the estimate for $\rho\left(\mathfrak{L}_{1}\right)$ can drastically affect the rate of convergence of the S.O.R. iterations and, thus, an accurate estimate of $\rho\left(\mathscr{L}_{1}\right)$ is needed.

In practice, two basic approaches are often used to estimate $\rho\left(\mathscr{L}_{1}\right)$ or equivalently $\omega_{b}$. One approach is to carry out a number of S.O.R. iterations with some $\omega<$ $\omega_{b}$ and then on the basis of numerical results obtain a new estimate for $\omega_{b}[1]$, [3], [4], [12]. A second approach is to obtain an estimate for $\rho\left(\mathfrak{L}_{1}\right)$, using the power method, prior to carrying out the S.O.R. iterations [1], [2], [5], [6]. The second approach is useful when the iterative process is slowly convergent, when the matrix problem (1.1) is to be solved a number of times for different vectors $f$, or when very accurate solutions are required.

In this paper it is shown how to use Chebyshev polynomials to accelerate the convergence of the power method. The description of the procedure includes a method for terminating the iterations in an attempt to minimize the total computer time required to solve the problem. The method has worked well in practice in a number of difficult problems. More details are given in [13].

The numerical procedures given in this paper apply equally well when the cyclic Chebyshev semi-iterative method [2], [7] is used to solve the matrix problem (1.1).

2. The Chebyshev Polynomial Method. By the S.O.R. method for solving (1.1) is meant the iterative process

$$
D \mathbf{x}^{n+1}=\omega\left\{L \mathbf{x}^{n+1}+U \mathbf{x}^{n}+\mathbf{f}\right\}+(1-\omega) D \mathbf{x}^{n}
$$

where $D$ is a block diagonal matrix and $L$ and $U$ are respectively strictly lower and upper triangular matrices. With the assumptions of Section 1, (2.1) converges most rapidly when $\omega=\omega_{b}$, where

$$
\omega_{b}=2 /\left\{1+\left[1-\rho\left(\mathfrak{L}_{1}\right)\right]^{1 / 2}\right\}
$$

and $\rho\left(\mathfrak{L}_{1}\right)$ is the spectral radius of the Gauss-Seidel matrix $\mathfrak{L}_{1}=(D-L)^{-1} U[2, \mathrm{p}$. 111]. Also, the eigenvalues $\lambda_{i}$ of the $q \times q$ matrix $\mathfrak{L}_{1}$ lie in the interval $[0,1)$ and may be ordered as

Received March 13, 1967. Revised August 1, 1967.

* Work performed while at Bettis Atomic Power Laboratory, Westinghouse Electric Corporation, West Mifflin, Pennsylvania. 


$$
1>\lambda_{1}=\lambda_{2}=\cdots=\lambda_{s-1}>\lambda_{s}=\lambda_{s+1}=\cdots=\lambda_{t-1}>\lambda_{t} \geqq \cdots \geqq \lambda_{q} \geqq 0,
$$

where we assume that $t>s$ [2, p. 107]. The ratio $\sigma=\lambda_{s} / \lambda_{1}$ is called the dominance ratio. We are concerned with finding $\lambda_{1}=\rho\left(\mathscr{L}_{1}\right)$.

In this section we assume that $\mathfrak{L}_{1}$ has $q$ linearly independent eigenvectors. Starting with a vector

$$
\mathbf{x}(0)=a_{1} \mathbf{u}_{1}+a_{s} \mathbf{u}_{s}+\sum_{i=t}^{q} a_{i} \mathbf{u}_{i}, \quad a_{1} \neq 0,
$$

where $\mathfrak{L}_{1} \mathbf{u}_{j}=\lambda_{j} \mathbf{u}_{j}$, we define

$$
\begin{aligned}
\mathbf{x}(r) & =P_{r}\left(\mathfrak{L}_{1}\right) \mathbf{x}(0) \\
& =P_{r}\left(\lambda_{1}\right)\left\{a_{1} \mathbf{u}_{1}+\frac{P_{r}\left(\lambda_{s}\right)}{P_{r}\left(\lambda_{1}\right)} a_{s} \mathbf{u}_{s}+\sum_{i=t}^{q} \frac{P_{r}\left(\lambda_{i}\right)}{P_{r}\left(\lambda_{1}\right)} a_{i} \mathbf{u}_{i}\right\}
\end{aligned}
$$

where $P_{r}$ is a polynomial of degree $r$ satisfying $P_{r}\left(\lambda_{1}\right) \neq 0$. If $P_{r}(y)=y^{r},(2.4)$ is the power method of iteration. In general $\mathbf{x}(r)$ approaches (in direction) the eigenvector $\mathrm{u}_{1}$ at a rate governed by $\max \left|P_{r}\left(\lambda_{i}\right) / P_{r}\left(\lambda_{1}\right)\right|, 2 \leqq i \leqq q$, if this maximum is $<1$. For the Chebyshev method we take $P_{r}(y)=P_{r, \sigma}(y)$, where

$$
P_{r, \sigma}(y)=\frac{T_{r}\left(2 y / \lambda_{1} \sigma-1\right)}{T_{r}(2 / \sigma-1)}
$$

and where $T_{r}(z)$ is the Chebyshev polynomial of degree $r$. Among all polynomials $P_{r}$ of degree $r$ in $y$ and such that $P_{r}\left(\lambda_{1}\right)=1, P_{r, \sigma}(y)$ is the polynomial having the least maximum modulus over the range $0 \leqq y \leqq \lambda_{s}$ [8]. For $\sigma$ close to unity, the Chebyshev polynomial method is much faster than the power method [9].

For computational purposes the vectors $\mathbf{x}(r)$ of the Chebyshev method can be formed in sequence by the procedure [10]

$$
\begin{aligned}
\mathbf{v}(r) & =\mathfrak{L}_{1} \mathbf{x}(r-1), \\
\lambda(r) & =\frac{[\mathbf{v}(r), \mathbf{x}(r-1)]}{[\mathbf{x}(r-1), \mathbf{x}(r-1)]} \\
\mathbf{x}(r) & =\mathbf{x}(r-1)+\alpha_{r}\left[\frac{\mathbf{v}(r)}{\lambda(r)}-\mathbf{x}(r-1)\right]+\beta_{r}[\mathbf{x}(r-1)-\mathbf{x}(r-2)],
\end{aligned}
$$

where $\alpha_{1}=2 /(2-\sigma), \beta_{1}=0$ and for $r \geqq 2$

$$
\alpha_{r}=\frac{4}{\sigma} \frac{T_{r-1}((2-\sigma) / \sigma)}{T_{r}((2-\sigma) / \sigma)}, \quad \beta_{r}=\frac{T_{r-2}((2-\sigma) / \sigma)}{T_{r}((2-\sigma) / \sigma)} .
$$

In the formula for $\lambda(r)$ the notation [ $\mathbf{v}, \mathbf{w}$ ] denotes the inner product of two vectors $\mathbf{v}$ and $\mathbf{w}$. We note that (2.6) reduces to the power method when $\alpha_{r}=1$ and $\beta_{r}=0$, $r=1,2, \cdots$. Also, since the matrix vector product $\mathfrak{L}_{1} \mathbf{x}(r-1)$ may be obtained using (2.1) by setting $\mathrm{f}=\mathrm{o}$ and $\omega=1$, the iterative procedure (2.6) takes essentially the same coding as that required by the S.O.R. iterations. However, (2.6) requires one and possibly two more vectors of storage than do the S.O.R. iterations. This could be troublesome in the solution of large problems. This will be discussed further in Section 3.

An accurate estimate for $\sigma$ is important in the effective use of the Chebyshev polynomial method. This problem is discussed in Section 4. 
3. Linear Elementary Divisors for $\mathfrak{L}_{1}$. In general, the elementary divisors of the Gauss-Seidel matrix are not all linear. However, if the $q \times q$ positive-definite matrix $A$ of (1.1) is partitioned in the "normal 2-cyclic" form [2, p. 101],

$$
A=\left[\begin{array}{ll}
A_{1} & B \\
B^{T} & A_{2}
\end{array}\right]
$$

then Tee [11] has shown that the Gauss-Seidel matrix associated with the partitioning (3.1) has only linear elementary divisors. (Another proof of this fact can be found in [13].) Thus, when $A$ is partitioned in the form (3.1), the Chebyshev procedure of (2.6) may be used to obtain an estimate for $\rho\left(\mathfrak{L}_{1}\right)$. However, when $A$ is of the form (3.1), one may use a different (and, perhaps, a better) Chebyshev procedure.

The Gauss-Seidel matrix $\mathfrak{L}_{1}$ associated with (3.1) may be written as

$$
\mathfrak{L}_{1}=\left[\begin{array}{ll}
A_{1} & 0 \\
B^{T} & A_{2}
\end{array}\right]^{-1}\left[\begin{array}{ll}
0 & -B \\
0 & 0
\end{array}\right]=\left[\begin{array}{ll}
0 & -A_{1}^{-1} B \\
0 & A_{2}^{-1} B^{T} A_{1}^{-1} B
\end{array}\right] .
$$

Thus, the nonzero eigenvalues of $\mathscr{L}_{1}$ are the same as those of $A_{2}^{-1} B^{T} A_{1}{ }^{-1} B$ and $\rho\left(\mathscr{L}_{1}\right)=\rho\left(A_{2}^{-1} B^{T} A_{1}^{-1} B\right)$. Also, the elementary divisors of $A_{2}^{-1} B^{T} A_{1}^{-1} B$ are linear, since the matrix $A_{2}^{-1} B^{T} A_{1}^{-1} B$ is similar to the symmetric matrix $A_{2}^{-1 / 2} B^{T} A_{1}^{-1}$ $B A_{2}{ }^{-1 / 2}$. Hence, the Chebyshev polynomial method may be applied to the matrix $A_{2}{ }^{-1} B^{T} A_{1}{ }^{-1} B$ and can be carried out by the procedure

$$
\begin{aligned}
& \hat{\mathbf{v}}(r)=A_{2}{ }^{-1} B^{T} A_{1}{ }^{-1} B \hat{\mathbf{x}}(r-1), \\
& \lambda(r)=\frac{[\hat{\mathbf{v}}(r), \hat{\mathbf{x}}(r-1)]}{[\hat{\mathbf{x}}(r-1), \hat{\mathbf{x}}(r-1)]} \\
& \hat{\mathbf{x}}(r)=\hat{\mathbf{x}}(r-1)+\alpha_{r}\left[\frac{\hat{\mathbf{v}}(r)}{\lambda(r)}-\hat{\mathbf{x}}(r-1)\right]+\beta_{r}[\hat{\mathbf{x}}(r-1)-\hat{\mathbf{x}}(r-2)],
\end{aligned}
$$

where $\alpha_{r}$ and $\beta_{r}$ are as defined in (2.6).

It is not necessary to form the matrix $A_{2}{ }^{-1} B^{T} A_{1}{ }^{-1} B$ explicitly in order to carry out (3.3). The vector $\hat{\mathbf{v}}(r)$ may be obtained implicitly by

$$
\begin{aligned}
& \hat{\mathbf{y}}(r)=A_{1}{ }^{-1} B \hat{\mathbf{x}}(r-1), \\
& \hat{\mathbf{v}}(r)=A_{2}{ }^{-1} B^{T} \hat{\mathbf{y}}(r) .
\end{aligned}
$$

Thus, to obtain $\hat{\mathbf{v}}(r)$ requires the same work as does the $\mathbf{v}(r)$ of (2.6). However, the eigenvalue and extrapolation calculations in (3.3) use vectors with fewer components than those used in (2.6). For example, if $A_{1}$ and $A_{2}$ are matrices of the same order, then the vectors $\mathbf{x}(r)$ of (2.6) contain twice as many components as do the $\hat{\mathbf{x}}(r)$ of (3.3). Thus, the arithmetic computations and the vector storage requirements of (3.3) are less than those of (2.6).

4. Computational Strategy and Numerical Results. In this section it is assumed that $A$ is partitioned in the normal 2-cyclic form (3.1). Thus, the Chebyshev iterative procedure (2.6) or (3.3) may be used to estimate $\rho\left(\mathfrak{L}_{1}\right)$. To complete the description of the Chebyshev algorithm, we shall (a) specify a means of estimating $\sigma$, (b) discuss the strategy of the algorithm, and (c) give a criterion for terminating the 
iterative procedure (2.6) or (3.3). Finally, we discuss some numerical results.

a. Estimating $\sigma$. Numerical estimates for $\sigma$ are obtained by observing the decay rate of the residual vector $\mathbf{y}(r) \equiv \mathbf{v}(r) / \lambda(r)-\mathbf{x}(r)$, where $\mathbf{v}(r), \lambda(r)$, and $\mathbf{x}(r)$ are defined by (2.6). We define the residual vector quotient as

$$
Q(r)=\|\mathbf{y}(r)\| /\|\mathbf{y}(r-1)\|,
$$

where $\|$ || denotes some suitable vector norm. Since for the power method, $Q(r) \rightarrow \sigma$ [13], an initial estimate for $\sigma$ may be obtained by doing a few power iterations and computing $Q(r)$ before starting the use of Chebyshev polynomials.

Estimates for $\sigma$ are obtained at every Chebyshev iteration by comparing the convergence rate actually being obtained with the theoretical convergence rate one would obtain if the $\sigma$ being used were correct. If a Chebyshev polynomial using $\sigma_{0}$ as the estimate for $\sigma$ is started on iteration $k+1$ and if

(i) $\lambda(k+r+1)$ is sufficiently close to $\lambda_{1}$ for all $r \geqq 0$, and

(ii) $\left\|\sum_{i=t}^{q} P_{r, \sigma_{0}}\left(\lambda_{i}\right) a_{i} \mathbf{u}_{i}\right\|$ is small relative to $\left\|P_{r, \sigma_{0}}\left(\lambda_{s}\right) a_{s} \mathbf{u}_{\varepsilon}\right\|$,

then from (2.4) and since $P_{r, \sigma_{0}}\left(\lambda_{1}\right)=1$, we may approximate $\mathbf{x}(k+r)$ by $a_{1} \mathbf{u}_{1}+$ $P_{r, \sigma_{0}}\left(\lambda_{s}\right) a_{s} \mathbf{u}_{s}$. Hence, the residual vector $\mathrm{y}(k+r+1)$ may be approximated by $\mathrm{y}(k+r+1) \approx(\sigma-1) P_{r, \sigma_{0}}\left(\lambda_{s}\right) a_{s} \mathbf{u}_{s}$ and the residual vector quotient by

$$
Q(k+r+1) \approx\left|P_{r, \sigma_{0}}\left(\lambda_{s}\right) / P_{r-1, \sigma_{0}}\left(\lambda_{s}\right)\right|,
$$

where $P_{0, \sigma_{0}}(y)=1.0$. With $Q_{r+1} \equiv \prod_{l-2}^{r+1} Q(k+l)$, it follows from (4.3) that

$$
Q_{r+1} \approx\left|P_{r, \sigma_{0}}\left(\lambda_{s}\right)\right| \text {. }
$$

Solving for the largest positive solution $\lambda_{s}$ of (4.4) and dividing by $\lambda_{1}$, one obtains the estimate*

$$
\sigma \approx \frac{\sigma_{0}}{2}\left\{\cosh \left[\frac{\cosh ^{-1}\left\{Q_{r+1} T_{r}\left(2 / \sigma_{0}-1\right)\right\}}{r}\right]+1.0\right\} .
$$

Thus, if the assumptions given in (4.2) are valid, expression (4.5) will give a good estimate for $\sigma$. Obviously, these assumptions do not always hold. However, they may be reasonable under certain conditions. The Chebyshev strategy given below is designed toward this end.

b. Chebyshev Strategy. Let the $k$ th iterate in the Chebyshev procedure be expressed in the form

$$
\mathbf{x}(k)=P_{r_{n}, \sigma_{n}}\left(\mathfrak{L}_{1}\right) \cdots P_{r_{1}, \sigma_{1}}\left(\mathfrak{L}_{1}\right) P_{r_{0}, \sigma_{0}}\left(\mathfrak{L}_{1}\right) \mathfrak{L}_{1}^{4} \mathbf{x}(0),
$$

where $P_{r, d}\left(\mathscr{L}_{1}\right)$ is the Chebyshev polynomial of degree $r$ in which $d$ is used as the estimate for $\sigma$ and where $k=4+r_{0}+\cdots+r_{n}$. Using the notation of (4.6), the Chebyshev strategy is first to do four iterations of the power type to obtain an initial estimate, $\sigma_{0}$, for $\sigma$ and a reasonable estimate for $\lambda_{1}$. In addition, these power iterates tend to eliminate from the eigenvector guess $\mathbf{x}(0)$ those eigenvector modes corresponding to small eigenvalues. Starting with iteration 5, low-degree Chebyshev polynomials are repeatedly applied, with the estimates for the dominance ratio being con-

* If $\left\{Q_{r+1} T_{r}\left(2 / \sigma_{0}-1\right)\right\}<1$, then cos and $\cos ^{-1}$ should be used in (4.5) instead of cosh and $\cosh ^{-1}$. 
tinuously updated. Upper limits of the form $\sigma_{0} \leqq .9, \sigma_{1} \leqq .95, \sigma_{2} \leqq .985$ are imposed on the first few estimates for $\sigma$ in an attempt to insure that these initial estimates be less than $\sigma$. Those polynomials for which $\sigma$ is underestimated will greatly reduce all the eigenvector modes in the estimated vector $\mathbf{x}(0)$ except those with the larger eigenvalues.* This results in assumption (ii) of (4.2) being more nearly satisfied which, in turn, enables the estimates for $\sigma$ to converge to the correct value. As $\sigma_{n}$ approaches $\sigma$, high-degree polynomials are applied.

The strategy for terminating a Chebyshev polynomial and starting the generation of a new polynomial using an improved estimate for $\sigma$ is made by comparing the convergence rate being obtained with the theoretical convergence rate one would obtain if the estimate for $\sigma$ were correct. If a Chebyshev polynomial using $\sigma_{0}$ as the estimate for $\sigma$ is started on iteration $k+1$, then from Eq. (4.3) the convergence rate actually being obtained on iteration $k+r$ is $-\ln [Q(k+r+1)]$. Now if $\sigma_{0}=\sigma$, the Chebyshev theory of Section 2 implies that the theoretical convergence rate of iteration $k+r$ should be $-\ln \left[T_{r-1}\left(2 / \sigma_{0}-1\right) / T_{r}\left(2 / \sigma_{0}-1\right)\right]$. Thus, the criterion

$$
R(k+r+1)=\frac{\ln [Q(k+r+1)]}{\ln \left[T_{r-1}\left(2 / \sigma_{0}-1\right) / T_{r}\left(2 / \sigma_{0}-1\right)\right]}<.6
$$

for starting the generation of a new polynomial automatically causes the degrees $r_{n}$ to become larger as $\sigma_{n}$ tends to $\sigma$. It is often helpful to require all polynomials generated to be at least of degree $r^{*}$, where $r^{*}$ is 3 or 4 .

c. Terminating the Iterative Procedure. Let $I_{\text {opt }}$ be the number of iterations required by the successive overrelaxation method using optimum $\omega$, and let $I_{\omega(k)}$ be the number of iterations required when

$$
\omega(k)=2 /\left\{1+(1-\lambda(k))^{1 / 2}\right\}
$$

is determined from the $k$ th approximation, $\lambda(k)$, of the $\lambda$ iterations. We wish to terminate the $\lambda$ iterations when $\lambda(k)$ is so close to $\rho\left(\mathscr{L}_{1}\right)$ that

$$
I_{\omega(k)} / I_{\text {opt }}-1<\delta \text {, }
$$

where $\delta$ is a number at our disposal. The quantity $\delta$ represents the relative number of additional S.O.R. iterations required because $\omega(k)$ rather than $\omega_{b}$ is used in (2.1).

In order to use (4.8) to terminate the $\lambda$ iterations, we must find some way to estimate $I_{\omega(k)}$ and $I_{\text {opt. }}$. Assuming that $\rho\left(\mathscr{L}_{1}\right) \approx 1$ and $\lambda(k) \approx \rho\left(\mathscr{L}_{1}\right)$ it is shown in [13] that (4.8) may be approximated by

$$
\begin{aligned}
& \frac{|\lambda(k)-\lambda(k-1)|}{2[1-\lambda(k)][1-Q(k)]}<\delta, \quad \lambda(k)>\rho\left(\mathfrak{L}_{1}\right), \\
& \left\{\frac{|\lambda(k)-\lambda(k-1)|}{[1-\lambda(k)][1-Q(k)]}\right\}^{1 / 2}<\delta, \quad \lambda(k)<\rho\left(\mathscr{L}_{1}\right) .
\end{aligned}
$$

Since in practice it usually happens that $\lambda(k)<\rho\left(\mathscr{L}_{1}\right)$, we terminate the iterations when (4.10) is satisfied.

The convergence quantity $\delta$ of (4.8) determines the total number of iterations required to solve the problem. To minimize computer time, it is desired to find the

* For example, if $\sigma=.889$ and if a 5 th-degree Chebyshev polynomial is generated with $\sigma_{0}=.8$, then all eigenvector modes $u_{i}$ with $\left(\lambda_{i} / \lambda_{1}\right) \leqq .8$ are multiplied by a factor smaller than .017 , while the $u_{s}$ eigenvector mode is multiplied by a factor of .211 . 
value of $\delta$ which minimizes this total number of iterations. In what follows we attempt to determine this optimum value experimentally.

d. Numerical Results. We now give two numerical examples which illustrate the effectiveness of the Chebyshev polynomial method. In each example $A$ is partitioned in the form (3.1). We take $\mathbf{f}=\mathbf{o}$ so that the solution of (1.1) is $\mathbf{x}=\mathbf{0}$. All components of the initial guess vector are taken to be 1.0 and the S.O.R. iterations are continued until the maximum vector component is less than $10^{-6}$. The $\lambda$ iterations performed prior to the S.O.R. iterations are carried out using the procedure given by (3.3) and the Chebyshev strategy is that as described in parts a and b of this section. For comparison purposes, the $\lambda$ iterations were also carried out using the power method.

Table 1. Problem A

Chebyshev Acceleration

\begin{tabular}{|c|c|c|c|c|c|c|}
\hline$k$ & $\lambda(k)$ & $\omega(k)$ & $\sigma(k)$ & $\delta(k)$ & $I_{\omega(k)}$ & $I_{T(k)}$ \\
\hline 5 & .997684 & 1.90816 & .89864 & .79 & $>5,000$ & $>5,000$ \\
\hline 33 & .999782 & 1.97091 & .99798 & .9 & 1,522 & 1,555 \\
\hline 49 & .999797 & 1.97189 & .99978 & 1.15 & 1,442 & 1,489 \\
\hline 70 & .999813 & 1.97302 & .99982 & .87 & 1,354 & 1,424 \\
\hline 82 & .999834 & 1.97453 & .99980 & .69 & 1,228 & 1,310 \\
\hline 103 & .999865 & 1.97706 & .99976 & .45 & 987 & 1,090 \\
\hline 115 & .999876 & 1.97798 & .99974 & .31 & 870 & 985 \\
\hline 130 & .999884 & 1.97869 & .99972 & .13 & 739 & 869 \\
\hline 132 & .999885 & 1.97874 & .99972 & .11 & 722 & 854 \\
\hline
\end{tabular}

Power Iterations

\begin{tabular}{|c|c|c|c|c|c|c|}
\hline$k$ & $\lambda(k)$ & $\omega(k)$ & $\sigma(k)$ & $\delta(k)$ & $I_{\omega(k)}$ & $I_{T(k)}$ \\
\hline 5 & .997684 & 1.90816 & .89864 & .75 & $>5,000$ & $>5,000$ \\
\hline 38 & .999190 & 1.94499 & .97795 & 1.00 & 3,322 & 3,360 \\
\hline 103 & .999674 & 1.96451 & .98529 & .76 & & \\
\hline 200 & .999782 & 1.97092 & .99802 & .88 & 1,522 & 1,722 \\
\hline 250 & .999793 & 1.97163 & .99934 & .98 & 1,465 & 1,715 \\
\hline 300 & .999797 & 1.97193 & .99970 & 1.02 & 1,441 & 1,741 \\
\hline
\end{tabular}

In Tables 1 and $2, k$ is the iteration index for the $\lambda$ iterations, $I_{\omega(k)}$ is the number of iterations required to solve (1.1) to the required degree of accuracy using $\omega(k)$ as the overrelaxation parameter, $I_{T(k)}=k+I_{\omega(k)}$, and $\delta(k)$ is the left-hand side of 
(4.10). A graph of $k$ vs $I_{T(k)}$ for problem B is given in Fig. 1. Table 3 clearly shows the advantage of the Chebyshev polynomial method over the power method.

Table 2. Problem B

Chebyshev Acceleration

\begin{tabular}{|c|c|c|c|c|c|c|}
\hline$k$ & $\lambda(k)$ & $\omega(k)$ & $\sigma(k)$ & $\delta(k)$ & $I_{\omega(k)}$ & $I_{T(k)}$ \\
\hline 5 & .995636 & 1.87607 & .88427 & .77 & 1,372 & 1,377 \\
\hline 13 & .998096 & 1.91639 & .97447 & .93 & 864 & 877 \\
\hline 24 & .998798 & 1.93298 & .99392 & .74 & 640 & 664 \\
\hline 40 & .998920 & 1.93638 & .99957 & 1.19 & 590 & 630 \\
\hline 64 & .999278 & 1.94768 & .99931 & .44 & 394 & 458 \\
\hline 78 & .999353 & 1.95041 & .99914 & .13 & 311 & 389 \\
\hline 80 & .999358 & 1.95058 & .99912 & .09 & 299 & 379 \\
\hline 120 & .999361 & 1.95068 & & .03 & 289 & 409 \\
\hline
\end{tabular}

\section{Power Iterations}

\begin{tabular}{|c|c|c|c|c|c|c|}
\hline$k$ & $\lambda(k)$ & $\omega(k)$ & $\sigma(k)$ & $\delta(k)$ & $I_{\omega(k)}$ & $I_{T(k)}$ \\
\hline 5 & .995636 & 1.87607 & .88427 & .77 & 1,372 & 1,377 \\
\hline 35 & .998140 & 1.91732 & .97724 & .81 & 854 & 889 \\
\hline 99 & .998789 & 1.93278 & .99559 & .77 & 642 & 741 \\
\hline 150 & .998882 & 1.93529 & .99899 & .97 & 608 & 758 \\
\hline 200 & .998922 & 1.93642 & .99954 & 1.12 & 588 & 788 \\
\hline 250 & .998950 & 1.93722 & .99961 & 1.12 & 576 & 826 \\
\hline 300 & .998974 & 1.93794 & .99958 & 1.06 & 565 & 865 \\
\hline
\end{tabular}

TABLE 3

\begin{tabular}{|c|c|c|}
\hline Problem & $\begin{array}{l}\text { Minim } \\
\text { Chebyshev } \\
\text { Method }\end{array}$ & $\begin{array}{l}I_{T(k)} \\
\quad \text { Power } \\
\text { Method }\end{array}$ \\
\hline A & 854 & 1,715 \\
\hline B & 379 & 741 \\
\hline
\end{tabular}




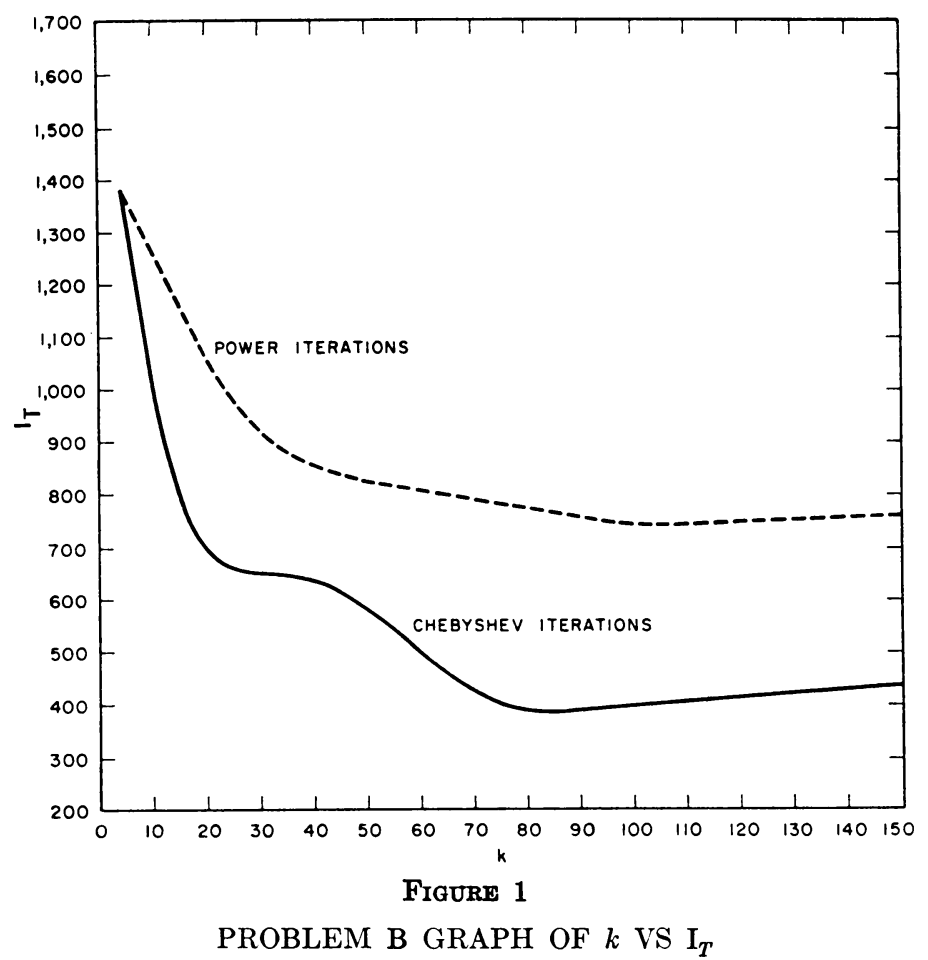

For the two problems considered here, $I_{T(k)}$ is minimized for the Chebyshev iterations when $\delta$ is about.1. Obviously, a different value for $\delta$ would minimize $I_{T(k)}$ for other problems. It has been found, however, that a convergence criterion for the $\lambda$ iterations of $\delta(k) \leqq .2$ works well for most problems.

Other numerical examples are given in [13].

e. Nonnormal Partitionings of $A$. It is sometimes more convenient from a programming point of view to use a 2-cyclic partitioning of $A$ which is not normal. For this case, however, the Chebyshev algorithm (2.6) usually requires many more iterations to estimate $\rho\left(£_{1}\right)$ accurately than would comparable problems with the normal partitioning (3.1). This is because $\aleph_{1}$ may have nonlinear elementary divisors corresponding to the eigenvalue 0 . In [13] there is presented a modification of the Chebyshev polynomial method which helps to overcome this difficulty.

Bettis Atomic Power Laboratory

Westinghouse Electric Corporation

West Mifflin, Pennsylvania

Institute for Fluid Dynamics and Applied Mathematics

The University of Maryland

College Park, Maryland

1. G. E. Forsythe \& W. R. Wasow, Finite-Difference Methods for Partial Differential Equations, Wiley, New York, 1960. MR 23 \#B3156. $28 \# 1725$.

2. R. S. VARGA, Matrix Iterative Analysis, Prentice-Hall, Englewood Cliffs, N. J., 1962. MR 
3. B. A. CARRE, "The determination of the optimum accelerating factor for successive overrelaxation," Comput. J., v. 4, 1961, pp. 73-78.

4. H. E. KULSRUD, "A practical technique for the determination of the optimum relaxation factor of the successive over-relaxation method," Comm. ACM, v. 4, 1961, pp. 184-187. MR 26 \#895.

5. A. K. RigLer, "Estimation of the successive over-relaxation factor," Math. Comp., v. 19, 1965, pp. 302-307. MR 31 \#5351.

6. E. L. WACHSPREss, Iterative Solution of Elliptic Systems and Applications to the Neutron Diffusion Equations of Reactor Physics, Prentice-Hall, Englewood Cliffs, N. J., 1966.

7. G. H. GoluB \& R. S. VARGA, "Chebyshev semi-iterative methods, successive overrelaxation iterative methods, and second order Richardson iterative methods. I, II," Numer. Math., v. 3, 1961, pp. 147-156, 157-168. MR 26 \#3207; MR 26 \#3208.

8. D. A. Flanders \& G. Shortley, "Numerical determination of fundamental modes," $J$. Appl. Phys., v. 21, 1950, pp. 1326-1332.

9. L. A. Hageman, The Chebyshev Polynomial Method of Iteration, WAPD-TM-537, 1967. (Available from the Clearinghouse for Federal Scientific and Technical Information, National Bureau of Standards, U. S. Department of Commerce, Springfield, Virginia.)

10. R. S. VARgA, Numerical Methods for Solving Multi-Dimensional Multigroup Diffusion Equations, Proc. Sympos. Appl. Math., Vol. 11, Amer. Math. Soc., Providence, R. I., 1961, pp. 164-189. MR 23 \#B595.

11. G. J. TEE, "Eigenvectors of the successive overrelaxation process, and its combination with Chebyshev semi-iteration," Comput. J., v. 6, 1963, pp. 250-263.

12. J. K. REID, "A method for finding the optimum successive overrelaxation parameter," Comput.J., v. 9, 1966, pp. 200-204. MR 33 \#3475.

13. L. A. Hageman \& R. B. Kellogg. Estimating Optimum Acceleration Parameters for Use in the Successive Overrelaxation and the Chebyshev Polynomial Methods of Iteration, WAPD-TM-592, 1966. (Available from the Clearinghouse for Federal Scientific and Technical Information; see reference 9.) 